\title{
ROBUSTNESS OF MULTIDIMENSIONAL OPTIMIZATION OUTCOMES: A GENERAL APPROACH AND A CASE STUDY
}

\author{
Negin Forouzesh \\ Layne T. Watson \\ Department of Computer Science \\ Virginia Polytechnic Institute and State University \\ Blacksburg, VA 24061
neginf@vt.edu \\ Department of Computer Science \\ Department of Mathematics \\ Department of Aerospace and Ocean Engineering \\ Center for Soft Matter and Biological Physics \\ Virginia Polytechnic Institute and State University \\ Blacksburg, VA 24061 \\ ltwatson@computer.org
}

\author{
Alexey V. Onufriev* \\ Department of Computer Science \\ Department of Physics \\ Center for Soft Matter and Biological Physics \\ Virginia Polytechnic Institute and State University \\ Blacksburg, VA 24061 \\ alexey@cs.vt.edu
}

\begin{abstract}
In multidimensional parameter optimization of complex systems, the preferred solution must also be robust to virtually inevitable perturbations and uncertainties. Having a conceptually simple and computationally facile metric that can help distinguish between candidate optimum solutions in a post processing step is useful. Motivated by free energy function in statistical physics, which evaluates a trade-off between entropy and enthalpy, here we introduce a novel statistical robustness metric that assesses robustness with respect to possible to inevitable uncertainties in the objective function values or optimal parameters. The metric is the expected value $\langle E\rangle$ of the objective function, evaluated using weighted samples in a box around each optimum. The width of the sample distribution is problem-specific. As a case study, the proposed robustness metric is employed to find the most robust optimal solution in 5-dimensional parameter space in the context of dielectric boundary optimization in atomistic modeling, relevant to computational drug discovery.
\end{abstract}

Keywords: robustness metric, multidimensional optimization, computational drug discovery.

\section{INTRODUCTION}

Global multidimensional optimization in real-world applications can be very difficult as the objective function landscape is often rugged with numerous local minimum points. It is not uncommon to identify several local optimum points that are hard to distinguish in practice - differences between the corresponding objec- 
tive function values may be too small to make the differences meaningful in the context of the underlying problem. What makes the problem even harder is that the evaluation of such an objective function at each search point can be computationally expensive. This computational expense makes it difficult to explore the objective function landscape at a fine grain resolution, often needed to identify the global optimum, see (He, Verstak, Watson, and Sosonkina 2009) and references there in.

As if finding a global optimum point was not hard enough, the problem of finding a practically useful optimum is even harder: a good solution must also be robust to unavoidable perturbations and uncertainties, virtually inevitable in any complex system being optimized. As an illustration, choosing the global optimum point, corresponding to the bottom of a deep but narrow well, may lead to undesirably high deviation from the optimum when the same solution is replicated with even a small uncertainty, or applied to a different data set, not used in the original optimization, Fig. 1. A way to address this issue is by identifying robust optimal parameters, which when subject to small perturbations around the optimum result in acceptable variations in the objective function (McPhail, Maier, Kwakkel, Giuliani, Castelletti, and Westra 2018). The robustness must be carefully balanced with the need to achieve the most optimal value of the objective function.

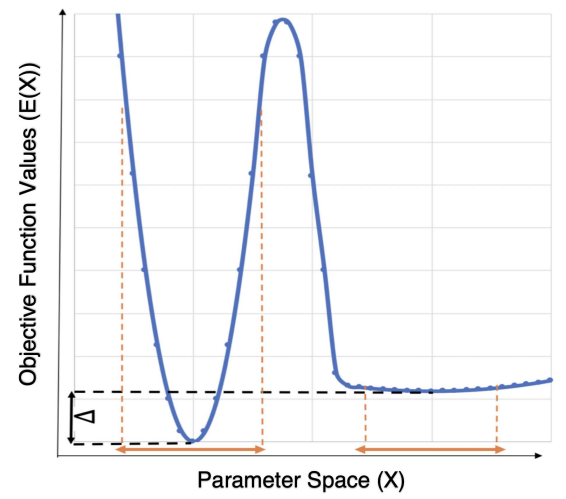

Figure 1: Depth vs. width trade-off in choosing the preferred optimum. The shallow and wide local minimum point on the right may be more robust to perturbation compared to the narrow but deeper global minimum solution on the left. When the difference $\Delta$ between the objective function at the minimum points is close to the problem-specific range in which two optima are practically indistinguishable, one may intuitively conclude that the wider local minimum solution should be preferred for a complex system with inevitable uncertainties in replication of its parameters. The goal of this work is to formulate and test a quantitative criterion for such preference.

Robust optimization is a field of optimization theory that aims to find optima under uncertainty. Assuming that modeling uncertainty with a deterministic function is possible, the initial objective function is modified into a more complex function which not only seeks to optimize the objective function but also minimizes the uncertainty. There are different approaches to implement this idea. For example, in (Kugele, Trosset, and Watson 2008) statistical decision theory is applied for quantifying uncertainties with applications in multidimensional integration. In (Zhou, Chaudhuri, Haftka, and Cheng 2014, Zhou, Haftka, and Cheng 2016), Zhou et al. introduced new algorithms to find competitive diverse alternatives to global optima. All of these approaches imply that enough detailed information about the uncertainty is available, so that it could be incorporated into the objective function directly as a deterministic component. However, note that incorporating the uncertainty directly into the objective function may limit the choice of optimization methods available for the specific problem. For hard multidimensional optimization problems this choice is already limited to begin with. 
A possible strategy to tackle the problem is to design a robustness metric that can be employed as a post processing step, after the optimization has been performed and the optima identified on the original objective function of the problem at hand. Such a metric decoupled from the objective function can also be not domain specific, and in principle could be easily applied to the outcome of any optimization resulting from the most appropriate method. Intuitively, the objective function near a robust optimum should not deviate sharply from the objective function value right at the optimum. In (Kwakkel, Eker, and Pruyt 2016) five statistical robustness metrics are introduced based on this idea: the distribution of the objective function values around a robust optimum is more skewed towards the value of that robust solution, which leaves little room for uncertainty about the expected outputs. These metrics solely investigate the robustness of the main objective function by studying the statistical characteristics of the points around the optima.

In this study, we go beyond the examination of the objective function exclusively found for a training set, and investigate the robustness of optima when their corresponding optimal solutions are utilized in new test cases, which has many practical applications. We propose a novel statistical robustness metric that addresses the depth vs. width trade-off (see Fig. 1), in a manner motivated by the similar concept in statistical physics: enthalpy-entropy competition in free energy function of thermodynamics. Here, we engineer the energy function to obtain a simple and general post processing robustness metric. Our robustness metric is evaluated through a case study: optimization of key parameters that define the molecular shape, in our case proteinligand (dielectric) boundary. Finding the most robust optimal solution in this problem not only improves the accuracy of these important practical calculations, but also guarantees approximately similar results when the optimized model is tested on new cases. This robustness is essential in drug discovery where binding free energy calculations play a key role. Since this work focuses on the robustness metric and not on the case study itself, we present only the minimal "bare bones" of the problem, just enough to illustrate our proposed metric.

\section{PROPOSED ROBUSTNESS METRIC}

\subsection{Motivating Example}

Given optimal parameters, in practice it is generally unlikely to replicate the exact optimal solution due to two major sources of error: first, new generated parameters may not be identical to the original optimal parameters. Second, optimal parameters are obtained based on a training data set. Although different strategies are typically incorporated to mitigate over fitting, it is virtually unavoidable that optimal parameters are not perfectly transferable to the same problem on another (test) data set. Therefore, we argue that a robust solution which leads to less error when a solution is replicated, may be preferred even if it is not the global optimal solution. The above reasoning about depth vs. width illustrated in Fig. 1 is intuitive but not easy to express in a mathematical form. The main difficulty is comparing the depth and the width on the same footing: in general, these are not even expressed in the same physical units, e.g., energy vs. length in the case of the optimization discussed in this work. Insight into a possible solution to the problem comes from statistical physics: free energy

$$
F=-\xi \ln \sum_{x} e^{-\left(E(x)-E_{g}\right) / \xi},
$$

includes both the depth (energy) and the width (entropy) of a state, where $E_{g}$ is the global minimum of the system's energy $E(x)$ and $\xi=k T$ is, in effect, the strength of the "thermal noise". The state $x$ with the lowest free energy $F$ corresponds to the most preferred thermodynamic state in the energy landscape $E(x)$ of the system. Unfortunately, Eq. (1) is derived for the specific case of physical systems in thermal equilibrium, and can not be assumed to be valid a priori for a general optimization problem. Moreover, it is not clear how to choose either "energy" or $\xi$ in Eq. (1) for a general system. For example, simply equating $E(x)$ in Eq. (1) with an objective function that corresponds to the cost of a car production is difficult to justify. Note that in 
physics $E(x)$ and $\xi$ have very specific properties that factor into the specific form of Eq. (1). Despite these conceptual difficulties, free-energy like functions have been used in machine learning (Shimazaki 2019) and optimization (Zhang, Saxe, Advani, and Lee 2018) mainly as the objective function. However, it is worth mentioning that even if the entire energy landscape is explored with a perfect objective function, finding the most robust solution is nontrivial and necessitates further analysis.

Hence in what follows a more general metric of robustness of optima is designed. Several observations about the structure of Eq. (1) give insights into the general structure of mathematical expressions that might be useful in comparing widths and depths of minima. The factor $e^{-\left(E(x)-E_{g}\right) / \xi}$ in Eq. (1) penalizes heavily all the contributions to the sum in $F$ that exceed the global minimum $E_{g}$ of $E(x)$ by more than $\xi$; the value of $\xi$ controls the penalty. In other words, only a few sample points contribute to the sum in $F$ from a narrow well, while many more contribute from a wide well. In the example of Fig. 1, the global minimum will be preferred if $\Delta \gg \xi$, while in the opposite case the higher local minimum will be preferred, if the corresponding well is wide enough.

\subsection{The Proposed Metric of Optima Robustness}

Inspired by the above example from statistical physics, the expected value $\langle E\rangle$ of the objective function taken over a neighborhood of the given optimum point is proposed as a measure of its robustness. Specifically, $\langle E\rangle=\int E(\mathbf{X}) P(\mathbf{X}) d \mathbf{X}$ where $P(\mathbf{X})$ is the probability distribution appropriate for the specific problem; $P(\mathbf{X})$ characterizes the uncertainty of reproducing the optimal parameters or the objective function in the vicinity of the optimum or both, see below. If $\left\langle E_{1}\right\rangle<\left\langle E_{2}\right\rangle$ then minimum point $\mathbf{X}_{\mathbf{1}}$ is preferred over minimum point $\mathbf{X}_{2}$, where $\left\langle E_{1}\right\rangle$ and $\left\langle E_{2}\right\rangle$ are the expected values of the objective function around minimum point $\mathbf{X}_{\mathbf{1}}$ and $\mathbf{X}_{2}$, respectively. Qualitatively speaking, $\langle E\rangle$ is a robustness metric compromising between "width" and "depth", Fig. 1. Motivated by the one-dimensional statistical discussion above, consider an exponentially decaying weighted sample in a box $B$ around a local minimum point $\mathbf{X}^{*}$ (in $n$ dimensions) given by

$$
\left\langle E \mid \mathbf{X}^{*}\right\rangle=\int_{B} E(\mathbf{X}) P(\mathbf{X}) d \mathbf{X}=\int_{B} E(\mathbf{X}) A e^{-(1 / 2)\left(\mathbf{X}-\mathbf{X}^{*}\right)^{t} \Sigma^{-1}\left(\mathbf{X}-\mathbf{X}^{*}\right)} d \mathbf{X},
$$

where $\Sigma$ is a $n \times n$ diagonal matrix with $\Sigma_{j j}$ being the empirical variance of $\mathbf{X}_{j}^{*}$, for $j \in 1, \ldots, n$. The specific form of $P(\mathbf{X})=A e^{-(1 / 2)\left(\mathbf{X}-\mathbf{X}^{*}\right)^{t} \Sigma^{-1}\left(\mathbf{X}-\mathbf{X}^{*}\right)}$, where $A$ is the normalization factor, is motivated by the ubiquity of Gaussian "noise" distributions in complex systems, where the noise comes from many independent sources that add up to produce an approximate Gaussian distribution. However, note that, in general, no statistical distributional assumptions have to be made here, and that any reasonable decaying weight function $P(\mathbf{X})$ based on the data could be used instead, as long as it satisfies the obvious normalization condition $\int_{B} P(\mathbf{X}) d \mathbf{X}=1$.

Next, we discuss specifics of the robustness metric on the two main sources of error considered earlier:

1. Error in the replication of the optimal parameters.

2. Deviation of the objective function for the training set used to find the optimal parameters from that for the test set. This can happen when the optimal parameters tailored for the primary training set are used on a new data set.

Without loss of generality and for the sake of simplicity and illustration, in what follows we consider $E(X)$ as a function of one variable $X$. In addition, for the sake of clarity and to simplify notation, in what follows we assume that the coordinate origin is shifted to $X^{*}$. 
Case I: uncertainty in reproducing parameters of the optimal solution. In a real-world complex system, exact replication of optimal parameters may be impossible due to multiple independent sources of error. Given the assumed normal distribution of optimal parameters, $X \sim \mathscr{N}\left(0, \hat{\sigma}^{2}\right)$, where $X=0$ is the initial optimal parameter and the mean of the distribution, and $\hat{\sigma}^{2}$ is the variance, it is straightforward to calculate the expected value of the objective function $E(X)$ on replicated optimal parameters:

$$
\langle E\rangle=A \int_{X \in \hat{B}} E(X) e^{-\frac{X^{2}}{2 \hat{\sigma}^{2}}} d X
$$

where $A$ normalizes the PDF and $\hat{B}$ is the sampling box around the minimum point $X=0$. Note the critical role of the width $\hat{\sigma}$ of the error distribution here: for very tight distributions, small $\hat{\sigma}$, Eq. (3) yields $\langle E\rangle \approx E(0)$, that is, the global minimum is identified as also the most robust. However, this is not the case in general, when $\hat{\sigma}$ is not small.

Case II: uncertainty in reproducing the objective function. Assume that the exact replication of optimal parameters is possible. (This is in fact the case in our application example considered in detail below, where the exact optimal atomic radii can be generated computationally). As discussed earlier, it is unavoidable that, when a new data set (test set) is considered, the objective function will deviate from that used in the training to find the optimal parameters. To measure this deviation, consider the shape of the objective function in the close vicinity of the optimal parameters. Around its minimum point on the training set, the objective function is (nearly) a parabola such as $E(X)=a X^{2}+c$. Deviation from this parabola results in another parabola such as $E^{\prime}(X)=a^{\prime}(X-b)^{2}+c^{\prime}$ on the test set. Note that shape conservation among all sets is a valid assumption because the training data set is supposed to be a legitimate representative of the whole set. In general, each new test set will have its own values of $a, b$ and $c$. However, note that the value of the objective function at its minimum point on each parabola is not affected by changes in " $a$ ". When several test data sets are studied, changes in " $c$ " lead to positive and negative deviations from the optimal objective function. It is not unreasonable to assume that this distribution is symmetric around its mean, and therefore the deviations in " $c$ " cancel out for a statistically significant number of test sets. Altogether, on average $E^{\prime}(0) \propto b^{2}$. Using a one-dimensional version of Eq. (2) for the illustration, $b \sim \mathscr{N}\left(0, \tilde{\sigma}^{2}\right)$. What the zero mean of the distribution implies is that the training set is well chosen, which is representative of the problem and unbiased. We assume this to be the case; the assumption can be verified explicitly in each specific case. Given this distribution, the average of the objective function values is

$$
\langle E\rangle \cong A \int_{b \in \tilde{B}} E(b) e^{-\frac{b^{2}}{2 \tilde{\sigma}^{2}}} d b,
$$

where $\tilde{B}$ is the sampling box around $b=0$, and $A$ normalizes the PDF. To estimate $\tilde{\sigma}$ in principle, one needs to compare $E^{k}(X)$ from a statistically significant number $k$ of independent test sets; each $E^{k}(X)$ is compared to $E(X)$ from the training set to identify the value of $b_{k}$, e.g., as in the example of Fig. 2 . Then, $\tilde{\sigma}$ is computed as a standard deviation of $b_{k}$.

Case III: general form of the proposed robustness analysis. In most cases, one can expect that one source of error will dominate. However, if both sources of error contribute roughly equally, then one needs to combine Eq. (3) and Eq. (4):

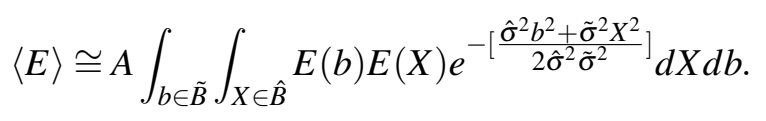




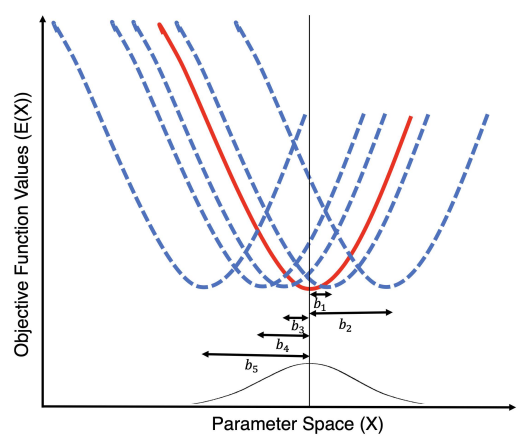

Figure 2: Estimation of the standard deviation of $b$ when several test cases are studied, as explained in Case II. Red solid parabola shows the optimum found on the training set, and dashed blue parabolas show the new optima when the optimal solution found for the training set is used in the test cases.

\subsection{Numerical Estimate of $\langle E\rangle$}

Here we estimate the expected value $\langle E\rangle=\int_{B} E(\mathbf{X}) P(\mathbf{X}) d \mathbf{X}$ of $E(\mathbf{X})$ over the box $B$ of volume $V(B)$, where $P(\mathbf{X})$ is the probability density function (PDF) of $\mathbf{X}$ in $B$ taken from Eq. (2). We sample $\mathbf{X}$ at $N$ points $\mathbf{X}_{i} \in B$ using the Latin hypercube sampling (McKay, Beckman, and Conover 1979), a common algorithm for high dimensions. With these sample points we approximate $\langle E\rangle \cong \frac{V(B)}{N} \sum_{i=1}^{N} E\left(\mathbf{X}_{i}\right) P\left(\mathbf{X}_{i}\right)$. The PDF is normalized so that $\frac{V(B)}{N} \sum_{i=1}^{N} P\left(\mathbf{X}_{i}\right)=1$, for random variables $\mathbf{X}$ (e.g., normal). We use $N=1000$ points everywhere, except for the purposes of testing convergence where we use $N=5000$. To find the size of the sampling box, the global minimum point was examined as follows: fix four of the five variables around this point alternatively, and change the fifth one so that the deviation from the optimal solution reaches $1.2 \mathrm{kcal} / \mathrm{mol}(\sim 2 \mathrm{kT})$. This strategy guarantees quite a wide region to gain meaningful samples, while avoiding potential overlaps between global and local solutions.

\subsection{Computational Cost}

To customize the proposed general robustness metric for a new domain, one needs to do the following calculations: (1) set a bounding box around each optimal solution (or optimum), (2) estimate the parameters of the distribution of the argument(s) of interest, (3) sample reasonably large number of points in the box(es), (4) calculate the expected value of the sample points given the probability density function found as the outcome of step (2). Note that these steps shall be executed only once. In our case study, the bottleneck of robustness calculations was the evaluation of objective function for 1,000 sample points. However, this cost was negligible compared to 40,000 evaluations of objective function performed by the global optimizer.

\section{CASE STUDY: ROBUST OPTIMAL ATOMIC RADII FOR MOLECULAR SIMULATIONS}

Drug discovery is one of the most challenging tasks in biological sciences as it takes about 10-15 years and \$1.5-2 billion on average to discover a new drug (Harrer, Shah, Antony, and Hu 2019). Therefore efforts to speedup this process or lower its costs are highly valuable. Computer-aided drug design (CADD) plays a crucial role in the early stage of drug discovery. In CADD, computational approaches are used in order to discover, develop, and analyze drugs and similar biologically active molecules, such as proteins. Proteins are an important class of biological macromolecules which perform their functionality mainly through 
interactions with other molecules, for example binding to small molecules so-called ligands (see Fig. 3). Several critical cellular processes such as gene expression and protein synthesis are controlled by proteinligand bindings as well as many biochemical reactions, which are catalyzed by special proteins- enzymes. A thorough understanding of protein-ligand interactions is central to comprehending biology at molecular level.

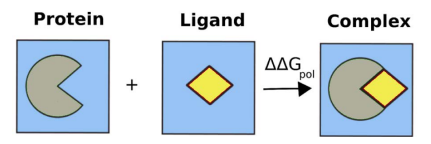

Figure 3: Protein-ligand binding process in water characterized by binding free energy, $\Delta \Delta G$. In this work, the main quantity of interest is the polar component of the binding free energy, $\Delta \Delta G_{p o l}$.

\subsection{A Note on Computing Binding Free Energy $(\Delta \Delta G)$}

Computational prediction of binding free energies is complex and challenging (Gilson and Zhou 2007), and its outcomes, including both accuracy and computational expense, can depend strongly on the underlying solvent model (Izadi, Harris, Fenley, and Onufriev 2018). Here we used GBNSR6 (Aguilar and Onufriev 2012) - a particular variant of the implicit solvation model (Onufriev 2010, Onufriev and Izadi 2018) that provides a relatively successful compromise between speed and accuracy, especially in the context of protein-ligand binding (Forouzesh, Izadi, and Onufriev 2017). Here we focus on the largest contribution to $\Delta \Delta G, \Delta \Delta G_{p o l}$, which can be computed directly by GBNSR6 given atomically-detailed structures of the protein, the ligand, and their complex, Fig. 3. The widely accepted accuracy goal (Gilson and Zhou 2007) for computational prediction of $\Delta \Delta G$ and its components is the so-called "chemical accuracy" of 1 $\mathrm{kacl} / \mathrm{mol}$.

\subsection{The Objective Function}

A key step in the implicit solvent modeling, used here for the calculation of the objective function, is the determination of the solute/solvent dielectric boundary (Onufriev and Aguilar 2014). The dielectric boundary is determined by the atomic radii of those comprising the protein surface $\left(\rho_{i}\right)$, as well as the radius of water probe $\left(\rho_{w}\right)$, Fig. 4 . In practice, admissible values of all the five radii $\left(\rho_{w}, \rho_{C}, \rho_{H}, \rho_{N}, \rho_{O}\right)$ are bonded from above and below; the specific bounds are unimportant in this illustrative example. There are no established "all-purpose best" values for these radii, which motivates the optimization problem.

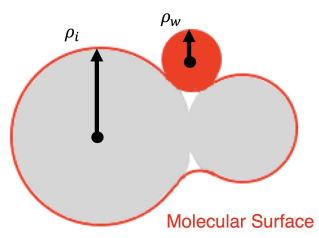

Figure 4: Solvent excluded surface (SES) exemplified for a "molecule" of two atoms. SES is shown as the red boundary, defined as the locus of the contact points (connected by circle arcs at contact discontinuities) of water probe (red circle) when it is rolled over the molecule (gray circles). The atomic radii $\left(\rho_{i}\right)$ and the water probe radius $\left(\rho_{w}\right)$ are simultaneously optimized, with $\rho_{i}+\rho_{w}$ constrained within its physical bounds.

Considering the five radii $\left(\rho_{w}, \rho_{C}, \rho_{H}, \rho_{N}, \rho_{O}\right)$ as free parameters, the dielectric boundary optimization turns to a multidimensional constrained optimization with respect to minimization of error in calculating $\Delta \Delta G_{p o l}$. 
The root-mean-square error (RMSE) objective function to be minimized is

$$
E_{C}(p)=\sqrt{\frac{1}{N} \sum_{c_{i} \in C}\left(\Delta \Delta G_{p o l}^{G B N S R 6}\left(c_{i}, p\right)-\Delta \Delta G_{p o l}^{T I P 3 P}\left(c_{i}\right)\right)^{2}},
$$

where $\Delta \Delta G_{\text {pol }}^{G B N S R 6}\left(c_{i}, p\right)$ is the polar binding free energy calculated by GBNSR6 for complex $\left(c_{i}\right)$ given point $p$ in the multidimensional parameter space of atomic radii $\left(\rho_{C}, \rho_{H}, \rho_{N}, \rho_{O}\right)$ and water probe radius $\left(\rho_{w}\right) . \Delta \Delta G_{\text {pol }}^{T I P 3 P}\left(c_{i}\right)$ is the reference polar binding free energy previously calculated, see below, for complex $\left(c_{i}\right)$, and $C$ is a given data set of $N$ complexes. The optimization is performed within the feasible range of the probe and atomic radii. The above objective function is deliberately cast in a form that retains the units (dimensionality) of the physical target quantity.

\subsection{Data sets}

The data set consists of 15 protein-ligand complexes for which reference $\Delta \Delta G_{p o l}$ were previously estimated (Izadi, Aguilar, and Onufriev 2015) in explicit solvent, TIP3P (Jorgensen, Chandrasekhar, Madura, Impey, and Klein 1983). The data set is partitioned into two subsets of eight (1pbk, $1 \mathrm{fkf}, 1 \mathrm{bkf}, 1 \mathrm{fkh}, 2 \mathrm{hah}$, 2fke, 1zp8, 1f40) and seven (1b11, 1fb7, 1fkb, 1fkg, 1fkj, 1fkl, 3kfp) complexes in order to train and test the proposed computational protocol, respectively. This partitioning guarantees a similar distribution of $\Delta \Delta G_{p o l}$ values between the two subsets.

\subsection{Optimization Method and Setting}

The deterministic DIRECT (Dividing Rectangles) global minimization algorithm (Jones, Perttunen, and Stuckman 1993) is a powerful optimization method for a moderate number of dimensions. DIRECT guarantees global convergence if the objective function is Lipschitz continuous, without requiring a gradient or knowledge of the Lipschitz constant. Using a massively parallel implementation of DIRECT, called VTDIRECT (He, Watson, and Sosonkina 2009), we introduce a computational pipeline to find the optimized atomic and water radii for binding free energy calculation of small protein-ligand complexes.

\subsection{Minima of the Objective Function}

VTDIRECT was employed for the 5-dimensional global optimization with respect to the objective function shown in Eq. (6), its argument being the vector of parameters: $\left(\rho_{w}, \rho_{C}, \rho_{H}, \rho_{N}, \rho_{O}\right)$. Critical to the success is the massively parallel nature of VTDIRECT95 that is efficiently implemented (He, Verstak, Watson, and Sosonkina 2009). Each round of minimization took 1.5 days using 64 CPUs (AMD Opteron(TM) Processor 6276 ) in parallel to run 40,000 objective function evaluations. VTDIRECT was set to return the best five minimum points (sorted based on the corresponding objective function values on the training set, $E_{\text {train }}$ ), separated by a distance of 0.5 unit using the Euclidean norm in 5-dimensional space. The results of this optimization are shown in Tab. 1. While the five optimal points shown in Tab. 1 are meaningfully far from each other in the 5-dimensional radii space, their objective function values on the training set $\left(E_{\text {train }}\right)$ are well within the range of $k T=0.6 \mathrm{kcal} / \mathrm{mol}$. That means that all of the five optima are well within the thermal noise of each other, and thus should be considered as practically indistinguishable with respect to the expected accuracy of $\Delta \Delta G_{p o l}$. However, as we have illustrated above, the depth of an optimum is not its only useful characteristic in the world of real, complex systems. In order to better differentiate these optima, we employ the proposed metric introduced in the previous section. The more robust optimum supposedly yields a lower $\langle E\rangle$. 
Table 1: Lowest five optimum parameter vectors found by VTDIRECT. Radii are in $\AA$ and units of the objective function value, $E$, are in $\mathrm{kcal} / \mathrm{mol}$.

\begin{tabular}{|l||l|l|l|l|l||l|}
\hline & $\rho_{W}$ & $\rho_{C}$ & $\rho_{H}$ & $\rho_{N}$ & $\rho_{O}$ & $E_{\text {train }}$ \\
\hline OPT 1 & 1.37 & 1.40 & 1.55 & 2.35 & 1.28 & 3.94 \\
\hline OPT 2 & 1.52 & 1.79 & 1.47 & 2.27 & 1.28 & 4.04 \\
\hline OPT 3 & 1.06 & 1.67 & 1.32 & 2.14 & 1.35 & 4.08 \\
\hline OPT 4 & 1.37 & 1.34 & 0.77 & 1.57 & 1.81 & 4.24 \\
\hline OPT 5 & 1.06 & 1.35 & 1.74 & 2.71 & 1.17 & 4.25 \\
\hline
\end{tabular}

\section{RESULTS AND DISCUSSION}

\subsection{Exploring the Objective Function Landscape}

To have better insight into the shape of the objective function landscape, the distribution of the objective function values around each minimum is examined on one thousand sample points in the close vicinity of the minimum point. In Fig. 5 boxplots along with outliers are illustrated with a clear depiction of quartile ranges. It is observed that OPT1, OPT2, OPT3, and OPT5 have less deviations in the sample boxes which implies "wide" shapes; whereas OPT4 shows more deviations from the corresponding minimum value, and is relatively more "narrow". As we shall see below, this observation, based on a fairly involved analysis, is well reflected in our proposed robustness metric.

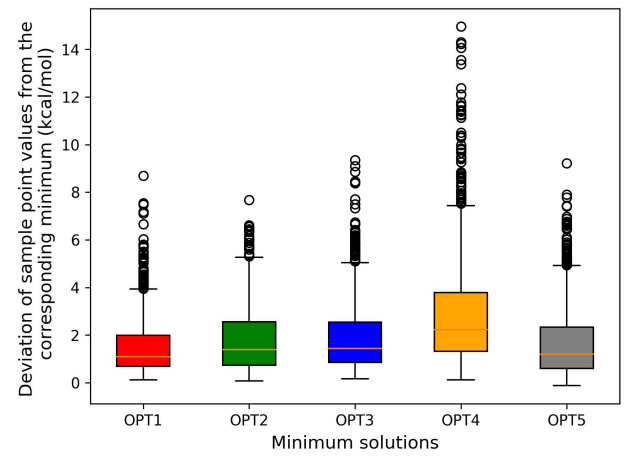

Figure 5: Boxplots of deviations in the objective function values around OPT1 (global minimum) and OPT2, OPT3, OPT4 and OPT5 (local minima) within the sampling box. Outliers are shown in circles.

\subsection{Robustness Analysis Based on the New Metric}

In the case study, it is possible to computationally replicate the exact five optimal parameters, and so our case is a pure Case II considered above. Hence, in what follows, a five dimensional form of Eq. (4) will be applied as the robustness metric for ranking the optimal solutions. The generalization of $\tilde{\sigma}$ in Eq. (4) is $\tilde{\Sigma}$ being the empirical variance the global optimal solution $\mathbf{X}^{*}$ from the seven test cases. Here $\tilde{\Sigma}$ is a fivedimensional diagonal matrix where $\operatorname{diag}(\tilde{\Sigma})=\left(\sigma_{W}, \sigma_{C}, \sigma_{H}, \sigma_{N}, \sigma_{O}\right)$, see Eq. (2). In other words, $\operatorname{diag}(\tilde{\Sigma})$ shows the standard deviation of each radius resulting form the use of possible new test cases. The initial test set introduced earlier is partitioned into seven test cases each made of one single protein-ligand complex. Thus, we are considering an instance of the general problem where one is interested in the performance of the optimal parameters on a single protein. As a result, we have a statistically meaningful distribution 
of $b$ values. Given seven test cases, the variance of $\mathbf{b}$ is calculated which finally results in $\operatorname{diag}(\tilde{\Sigma})=$ $(0.16,0.12,0.05,0.18,0.03)$. We apply the same $\tilde{\Sigma}$ to evaluate robustness of all the optima in Tab. $2-$ the use of the same $\tilde{\Sigma}$ is justified by the assumption that the overall shape of the test set objective function is similar to that of the training set. Objective function values $E_{\text {train }}$ and the robustness metric values $\left\langle E_{\text {train }}\right\rangle$ for the best five optimal solutions, OPT1 to OPT5, are shown in Tab. 2. While all the $E_{\text {train }}$ values are within the $k T$ range, the proposed robustness metric $\langle E\rangle$ accentuates the difference between the optima. This is particularly clear when OPT1 and OPT4 optima are compared: while the $E_{\text {train }}$ values of these two optima are very similar, the $\left\langle E_{\text {train }}\right\rangle$ values are meaningfully $(1 \mathrm{kcal} / \mathrm{mol})$ different. In fact, it turns out that OPT1 and OPT2 are the most robust optima, and OPT4 is the least robust optimum. The ranking based on $\langle E\rangle$ is conserved among 1000 and 5000 sampling scenarios, which supports the convergence of the method: the maximum relative difference between the two scenarios did not exceed $0.6 \%$.

To validate the above conclusions based on the new metric, the robustness of the five optima is further evaluated on the test set, see the last two columns of Tab. 2. Here, $E_{\text {test (combined) }}$ shows the RMSE of $\Delta \Delta G_{\text {pol }}$ calculations for the whole seven test cases in the test set. Consistent with the $\left\langle E_{\text {train }}\right\rangle$ ranking, OPT4 with the worst ranking based on $E_{\text {train }}$ also has the most deviation from its optimum based on $E_{\text {test }(\text { combined })}$. Also, OPT2 and OPT1 have the best $\left\langle E_{\text {train }}\right\rangle$ rankings, and these show the best performance based on MAX $\left(E_{\text {test }}\right.$ (single $)$. The other four optima are in the range of $(6.61-5.96 \mathrm{kcal} / \mathrm{mol} \approx 0.6 \mathrm{kcal} / \mathrm{mol}=\mathrm{kT})$ when employed on the test set. We also investigated the maximum deviation from the reference, among all the test cases, see the last column in Tab. 2. Aligned with our robustness ranking, OPT1 has one of the lowest maximum relative deviations while OPT4 has the highest maximum relative deviation. Altogether, it is concluded that the ranking based on $\left\langle E_{\text {train }}\right\rangle$ is consistent with further, more detailed robustness analysis based on the entire test set and its individual elements. This consistency validates the proposed metric.

Table 2: Robustness analysis of the lowest five optima found by VTDIRECT. Units of $E$ are in $\mathrm{kcal} / \mathrm{mol}$. $E_{\text {train }}$ is the objective function value on the training set, $\left\langle E_{\text {train }}\right\rangle$ is the robustness ranking on the training set, $E_{\text {test (combined })}$ is the objective function value on all of the test set elements combined, and $M A X\left(E_{\text {test }(\text { single })}\right)$ is the maximum relative deviation in calculating $\Delta \Delta G_{p o l}$ for each single test case compared to the reference.

\begin{tabular}{|l||l|l|l|l|}
\hline & $E_{\text {train }}$ & $\left\langle E_{\text {train }}\right\rangle$ & $E_{\text {test }}($ combined $)$ & $M A X\left(E_{\text {test }}\right.$ (single $)$ \\
\hline OPT 1 & 3.94 & 4.73 & 6.61 & $85 \%$ \\
\hline OPT 2 & 4.04 & 4.75 & 6.33 & $65 \%$ \\
\hline OPT 3 & 4.08 & 5.00 & 6.09 & $92 \%$ \\
\hline OPT 4 & 4.24 & 5.75 & 7.87 & $147 \%$ \\
\hline OPT 5 & 4.25 & 4.87 & 5.95 & $90 \%$ \\
\hline
\end{tabular}

\section{CONCLUSION}

In this study, we introduced a novel statistical robustness metric generally applicable as a post processing analysis tool to outcomes of optimization methods. The proposed metric is the expected value $\langle E\rangle$ of the objective function, evaluated using weighted samples around each optimum. Motivated by free energy function in statistical physics, yet independent from uncertainties of how to choose "energy" and "thermal noise" in the context of a general optimization exercise unrelated to statistical physics, our metric assesses robustness with respect to unavoidable uncertainties in the objective function values as well as optimal parameters. Similar underlying concept cuts across multiple disciplines; for example in (Forouzesh, Kazemi, and Mohades 2014) von Neumann entropy was used as a measure of the complexity of a system. Here, our focus is on robustness of the optimal solution, critical in many real-world applications. The example we have chosen to illustrate the novel approach here is optimization of atomic radii that directly affect the accuracy of free energy calculations in protein-ligand binding. The proposed robustness metric was applied 
on the lowest five practically indistinguishable optima found by our choice of optimization method, called VTDIRECT: the metric helped to clearly distinguish several optima otherwise indistinguishable. Further analysis confirmed that the most robust optima found by our metric are indeed those that deviate less from their corresponding optimal values when multiple test cases, independent of the training set, were evaluated. At the same time, the new metric also helped us identify the least robust optimum very clearly. We expect that, long-term, the most robust optimum radii identified in the context of protein-ligand binding will help improve the transferrability of these optimal settings across various types of relevant practical simulations and drug candidates (ligands). We emphasize that the only goal of this work was to introduce the new metric, and details of the case study are outside of its scope and will be published elsewhere. The proposed metric is general, and we expect it to be potentially useful in many other fields. For example, it could be used to check the convergence of deep neural networks and eliminate local non-robust solutions. The application of the new metric in practice requires essentially the knowledge of just one parameter - the variance of the most significant "noise" affecting optimal parameter replication. In the future, each field may be able to develop its own intuition for the magnitude of the variance, which would simplify the technicality of next applications of the proposed approach.

\section{ACKNOWLEDGMENTS}

The authors acknowledge Advanced Research Computing at Virginia Tech for providing computational resources and technical support. This work was supported by the NIH R21 GM131228 to A.V.O.

\section{REFERENCES}

Aguilar, B., and A. V. Onufriev. 2012. "Efficient computation of the total solvation energy of small molecules via the R6 generalized Born Model". Journal of chemical theory and computation vol. 8 (7), pp. 24042411.

Forouzesh, N., S. Izadi, and A. V. Onufriev. 2017. "Grid-based surface generalized Born model for calculation of electrostatic binding free energies". Journal of chemical information and modeling vol. 57 (10), pp. 2505-2513.

Forouzesh, N., M. R. Kazemi, and A. Mohades. 2014. "Structure-Based Analysis of Protein Binding Pockets Using Von Neumann Entropy". pp. 301-309. Springer.

Gilson, M. K., and H. X. Zhou. 2007. "Calculation of protein-ligand binding affinities". Annu. Rev. Biophys. Biomol. Struct. vol. 36, pp. 21-42.

Harrer, S., P. Shah, B. Antony, and J. Hu. 2019. “Artificial intelligence for clinical trial design”. Trends in pharmacological sciences.

He, J., A. Verstak, L. T. Watson, and M. Sosonkina. 2009. "Performance modeling and analysis of a massively parallel DIRECT : Part 1". The International Journal of High Performance Computing Applications vol. 23 (1), pp. 14-28.

He, J., L. T. Watson, and M. Sosonkina. 2009. "Algorithm 897: VTDIRECT95: serial and parallel codes for the global optimization algorithm DIRECT". ACM Transactions on Mathematical Software (TOMS) vol. 36 (3), pp. 1-24.

Izadi, S., B. Aguilar, and A. V. Onufriev. 2015. "Protein-Ligand Electrostatic Binding Free Energies from Explicit and Implicit Solvation”. Journal of chemical theory and computation vol. 11 (9), pp. 44504459.

Izadi, S., R. C. Harris, M. O. Fenley, and A. V. Onufriev. 2018. "Accuracy comparison of generalized Born models in the calculation of electrostatic binding free energies". Journal of chemical theory and computation vol. 14 (3), pp. 1656-1670. 
Jones, D. R., C. D. Perttunen, and B. E. Stuckman. 1993. "Lipschitzian optimization without the Lipschitz constant". Journal of Optimization Theory and Applications vol. 79 (1), pp. 157-181.

Jorgensen, W. L., J. Chandrasekhar, J. D. Madura, R. W. Impey, and M. L. Klein. 1983. "Comparison of simple potential functions for simulating liquid water". The Journal of chemical physics vol. 79 (2), pp. 926-935.

Kugele, S., M. Trosset, and L. Watson. 2008. "Numerical integration in statistical decision-theoretic methods for robust design optimization". Structural and Multidisciplinary Optimization vol. 36 (5), pp. 457-475.

Kwakkel, J. H., S. Eker, and E. Pruyt. 2016. "How robust is a robust Policy? Comparing alternative robustness metrics for robust decision-making”. In Robustness Analysis in Decision Aiding, Optimization, and Analytics, pp. 221-237. Springer.

McKay, M. D., R. J. Beckman, and W. J. Conover. 1979. "Comparison of three methods for selecting values of input variables in the analysis of output from a computer code". Technometrics vol. 21 (2), pp. 239245.

McPhail, C., H. Maier, J. Kwakkel, M. Giuliani, A. Castelletti, and S. Westra. 2018. "Robustness metrics: How are they calculated, when should they be used and why do they give different results?". Earth's Future vol. 6 (2), pp. 169-191.

Onufriev, A. 2010. "Continuum Electrostatics Solvent Modeling with the Generalized Born Model". In Modeling Solvent Environments, edited by M. Feig, pp. 127-165. USA, Wiley. Chapter: public policy not applicable.

Onufriev, A. V., and B. Aguilar. 2014. "Accuracy of continuum electrostatic calculations based on three common dielectric boundary definitions". Journal of Theoretical and Computational Chemistry vol. 13 (03), pp. 1440006.

Onufriev, A. V., and S. Izadi. 2018. "Water models for biomolecular simulations". Wiley Interdisciplinary Reviews: Computational Molecular Science vol. 8 (2), pp. e1347.

Shimazaki, H. 2019. "The principles of adaptation in organisms and machines I: machine learning, information theory, and thermodynamics". arXiv preprint arXiv:1902.11233.

Zhang, Y., A. M. Saxe, M. S. Advani, and A. A. Lee. 2018. "Energy-entropy competition and the effectiveness of stochastic gradient descent in machine learning". Molecular Physics vol. 116 (21-22), pp. 3214-3223.

Zhou, Y., A. Chaudhuri, R. T. Haftka, and G. Cheng. 2014. "Global search for diverse competitive designs". In 15th AIAA/ISSMO Multidisciplinary Analysis and Optimization Conference, pp. 24-39.

Zhou, Y., R. T. Haftka, and G. Cheng. 2016. "Balancing diversity and performance in global optimization". Structural and Multidisciplinary Optimization vol. 54 (4), pp. 1093-1105.

\section{AUTHOR BIOGRAPHIES}

NEGIN FOROUZESH is a Ph.D. candidate in computer science at Virginia Tech. She has interests in computational biology and bioinformatics. Her email address is neginf@vt.edu.

LAYNE T. WATSON (Ph.D., University of Michigan, 1974) has interests in numerical analysis, mathematical programming, bioinformatics, and data science. His email address is ltwatson@ computer.org.

ALEXEY V. ONUFRIEV (Ph.D., Brown University, 1997) has interests in computational and theoretical molecular biophysics. His email address is alexey@cs.vt.edu. 